\title{
The Profile Countermovement Jump for Kick Boxing Athletes
}

\author{
Muhammad Reza Aziz Prasetya ${ }^{1, *}$, Awang Firmansyah ${ }^{1}$, Dede Rahman ${ }^{1}$, Jeffry \\ Oktavian $^{1}$, Asep Aziz ${ }^{2}$
}

\author{
${ }^{1}$ Universitas Negeri Surabaya, Indonesia \\ ${ }^{2}$ KineticX, Indonesia \\ *Corresponding author. Email: muhammadreza.18058@mhs.unesa.ac.id
}

\begin{abstract}
The countermovement leap (C.M.J.) is described as a easy, practical, legitimate, and exceptionally dependable degree of lower frame energy. C.M.J. is the maximum dependable degree of lower frame strength compared to different leaping assessments. The C.M.J. has also been shown to be associated with dash overall performance, maximal electricity, and explosive power exams. This type of research is descriptive studies. The superior selection in this study was based on research that wanted to examine and see the countermovement jump (C.M.J.) test by kickboxing athletes. The purpose of this study is to investigate and see the results of lower body strength using the countermovement jump (C.M.J.) test, which contains components in the C.M.J. test including jump height, power, concentric force, eccentric force, which is carried out by kickboxing athletes. The results showed the maximum value of each test component, namely jump height 43.7, power 4280, Concentric force 1155, eccentric force 789 . Research on lower body strength conducted by kickboxing athletes or the so-called Countermovement Jump has proven reliable compared to other jumping tests. The C.M.J. can therefore be a suitable test for athletes participating in sports that require high levels of explosive power.
\end{abstract}

Keywords: Countermovement jump, Kick boxing, Lower body.

\section{INTRODUCTION}

The countermovement jump (C.M.J.) is defined as a simple, practical, legitimate, and extraordinarily reliable measure of decrease frame strength. As a result, it is no marvel that it has come to be a cornerstone take a look at for many electricity and conditioning coaches and sports scientists. The C.M.J. is the most reliable degree of decrease body electricity in comparison to other leaping checks. The C.M.J. has also been proven to be associated with sprint overall performance, maximal strength, and explosive power checks [1]. The countermovement soar is likewise a vertical jump and one of the maximum frequently used assessments for a maximal anaerobic strength output of the decrease extremities. it is an eccentric-concentric muscle movement that entails a coordinated extension of the trunk, hips, knees, and ankles [2].

The countermovement soar (C.M.J.) is primarily used to degree the explosive energy of an athlete's lower frame. It has turn out to be one of the maximum regularly used exams by coaches and researchers to measure electricity within the lower limbs [3] indirectly. considering the fact that power is a important issue in many sports activities, and the C.M.J. is a easy, practical, and dependable measure of strength inside the lower limbs, it would look like an obvious preference as a tool for measuring and monitoring performance. but, further research is wanted to quantify the relative contribution of the kinetic and kinematic variables to this motion. From the kinetic determinants, a greater pressure or torque development price is frequently related to progressed C.M.J. performance [1]. Approximately one-third of the performance improvement consequences in arm swing from paintings and power precipitated in the shoulder joint. Countermovement intensity has additionally been related to C.M.J.'s performance, growing the variety of movement of the knee joint from $70^{\circ}$ to $90^{\circ}$ resulting in a $17 \%$ growth in C.M.J.'s top. in addition, a excessive ankle dorsiflexion range of motion has been shown to contribute to C.M.J. performance in men however now not women [4].

Latest research have proven that comprehensive perception into an athlete's neuromuscular feature can be acquired thru distinct analysis of force-time curves throughout specific levels or the C.M.J. when as compared to measuring soar output alone. consequently the C.M.J. can be a appropriate take a look at for athletes 
taking part in sports activities that require a high degree of explosive energy, including soccer, rugby, basketball, weightlifting, and volleyball. similarly, this check has also been shown to be the most reliable measure of lower frame strength in comparison to different popular jumping tests. therefore, it is crucial for practitioners wishing to apply a resilience platform-primarily based C.M.J. assessment to recognize the constituent components of the C.M.J. force-time curve and recognize their relative contribution to C.M.J. overall performance and how they may be manipulated through training and education [5].

Several investigators have compared the kinetic and kinematic determinants of C.M.J., the kinematic technique factor being much less important than muscle capability. but, their technical evaluation is limited to the timing of the location of the lowest vertical middle of mass and using the swingarm. An boom in strength does no longer necessarily bring about a subsequent overall performance improvement [6].

Several researchers have found C.M.J. performance to be an objective marker of fatigue and supercompensation. However, other investigators have reported mixed results using C.M.J. measurements [12]. There are differences in findings that can be attributed to the combination of common and specific factor genes. Which includes general factors, namely: population type, duration or intensity, and intensity of activities carried out can be formed [7]. Even as the particular factors included are reporting many specific kinematic and kinetic variables, as an example, height, height electricity, relative height energy, relative electricity, common energy, peak pace, peak force, common force, charge of improvement of pressure, extrinsic time. /concentric time, flight time/eccentric time. And flight time/contraction time without or with C.M.J. load [8].
Based on the explanation above, the countermovement jump (C.M.J.) test has usually performed a function obtained through detailed analysis of the force-time curve across the specific phases of the C.M.J., beyond jump height alone [9]. The primary research revealed that when the C.M.J. variable was averaged across all trials conducted.

\section{METHODS}

This type of studies is descriptive research. The illustrative technique may be interpreted as a hasslefixing process investigated by means of describing the country of the problem or object within the examine, which may be inside the form of people, establishments, communities, and others primarily based on visible or information.

The descriptive selection in this study was based on research that wanted to examine and see the countermovement jump (C.M.J.) test by kickboxing athletes. This research instrument uses a force desk test, then tested using a countermovement jump test. Analysis of the data using descriptive, the test procedure was carried out at the Rumble TC venue with 18 kickboxing recreation teams, 13 men and five women, no injuries, and recreational couples. The countermovement jump variables are jump height, power, eccentric and concentric.

\section{RESULTS AND DISCUSSION}

The following are some of the research results that have been carried out, including the results of individual and overall tests.

Table 1. Test countermovement jump

\begin{tabular}{|l|c|c|c|c|c|c|c|}
\hline \multicolumn{1}{|c|}{ Comp. Test } & N & Range & Min. & Max & Mean & Std. Deviation & Variance \\
\hline Jump Height & 18 & 29.4 & 14.3 & 43.7 & 24.935 & 8.3019 & 68.921 \\
\hline Power & 18 & 2685 & 1595 & 4280 & 2795.22 & 704.652 & 496534.654 \\
\hline Concentric Force & 18 & 634 & 521 & 1155 & 677.44 & 149.601 & 22380.497 \\
\hline Eccentric Force & 18 & 1017 & 789 & 1806 & 1187.94 & 250.347 & 62673.585 \\
\hline Valid N (listwise) & 18 & & & & & & \\
\hline
\end{tabular}

Based on the data analysis above, the following are the results of Jump Height (J.H.), Power (P), Concentric Force (C.F.), and Eccentric Force (E.F.), as follows, the amount of data in this study is 18 athletes Kick Boxing Rumble TC. The maximum value of J.H. is $43.7 \mathrm{~cm}$, while the minimum value of J.H. is $14.3 \mathrm{~cm}$. The range of $\mathrm{J.H}$. is known to be $29.4 \mathrm{~cm}$, and the mean obtained is $24.935 \mathrm{~cm}$. Then the J.H. standard deviation is 8.3019 $\mathrm{cm}$, and the J.H. variance is 68.921. Furthermore, the maximum value of $\mathrm{P}$ is $4280 \mathrm{~W}$ with a minimum value of $\mathrm{P}$, which is $1595 \mathrm{~W}$. The obtained Range $\mathrm{P}$ is $2685 \mathrm{~W}$, and the mean is known to be $2795.22 \mathrm{~W}$. The standard deviation of $\mathrm{P}$ is $704.652 \mathrm{~W}$, and the variance of $\mathrm{P}$ is 496534,654. For Concentric force (C.F.) results, the minimum value obtained is 521 , and the maximum is 1155 with a standard deviation of 149,601 with a variation of 22380,497 . The results of the average Concentric force of 677.44 with a range of 634 . For the 
eccentric strength (E.F.) results, the minimum value obtained is 789, and the maximum is 1806 . While for the Standard deviation, the value is 250,347 with a variance of 62673,585 , and for the results of the average eccentric force of 1187.94 with a range of 1017 .

The results showed the maximum value of each test component, namely jump height 43.7, power 4280, Concentric force 1155 , eccentric force 789 . While the mean jump height is 24,935, Power is 2795.22, concentric force is 677.44 , and the strange point is 1187.94. The minimum jump height is 14.3 , the power is 1595 , the concentric force is 521 , and the eccentric point is 789. For the range of the jump height, 29.4, the emphasis is 2685 , the concentric force is 634 , and the abnormal staff is 1017 . For the standard deviation, the result is from jump height of 8,3019 , power of 704,652, the concentric force of 149,601, and eccentric force of 250,347. Meanwhile, the variance resulting from the jump height is 68,921 , for the power is 496534,654 , the concentric point is 22380,497 , and the eccentric force is 62673,585 .

Discussion tracking neuromuscular fame aligns with the capacity of the C.M.J. to come across the effects of fatigue and splendid-reimbursement on overall performance. The concept of neuromuscular popularity turned into followed in this observe, the usage of C.M.J. performance to screen fatigue and/or splendid compensatory consequences. All comparisons between the highest and average C.M.J. Overall performance discovered the mean rating extra touchy than the best rating in tracking neuromuscular popularity [13]. In addition, the following C.M.J. performance variables were additionally taken into consideration appropriate to evaluate the outstanding-compensation effect after the training intervention: top energy, common strength, height velocity, peak pressure, common impulse, and power calculated by way of equation [14]. The number one evaluation found out that after the C.M.J. variable was averaged across all trials performed, the sensitivity of the variable to discover changes in overall performance was progressed in comparison to reporting the very best price. This suggests that the possibilities of locating the real score growth whilst the imply is used to tune changes in C.M.J. performance [10].

Using the imply fee is generally used for the biomechanical parameter and has been counseled for the relevant physiological parameters. based on the modern findings, we could not decide whether there was a distinction within the sensitivity of the C.M.J. variables related to the range of C.M.J. trials averaged. This C.M.J. overall performance evaluation has used a metaanalytical method to determine and justify why the use of imply values is greater sensitive than the best rankings in detecting exchange [15]. but, psychological, physiological, and biomechanical elements also play an vital function within the overall performance of the C.M.J. in phrases of biological variability. The psychological effect of direct know-how of the consequences at some stage in multiple trials contributes to the reliability of the measurements. different influential elements may also encompass; (i) changes inside the excitation-contraction coupling procedure and (ii) musculoskeletal redundancy, that is defined as fluctuations in individual muscle interest during pressure production that cause variability in muscle activation styles [11]. consequently, the natural variability of overall performance in the course of human movement can be addressed. properly, whilst the mean method is used to reveal neuromuscular reputation. Acute look at exclusion (3 weeks in duration) can also have affected the outcomes. critical research had been excluded to minimize confounding of education interventions (shorttime period and long-term); however, the course of the blanketed interventions varied extensively and need to be considered whilst interpreting the results [24]. Of be aware, many acute interventions have located the C.M.J. timing variable to be sensitive in detecting critical changes in C.M.J. performance.

Appreciably, the functionality to lessen the diploma of muscle slack and speedy build up stimulation in the S.J. can be important to high-depth sports activities overall performance due to the fact there's usually now not sufficient time to perform a large countermovement at some stage in maximum excessive-depth sports situations [16]. Schooling would possibly consequently try and decrease the difference among C.M.J. and S.J. performances by training in particular on these talents. As said previously, the capability to quickly building up stimulation may be associated with coordination. it's miles critical to precisely mimic the intermuscular coordination styles of vertical jumping in the course of training [17]. Schooling would possibly consequently try and decrease the difference among C.M.J. and S.J. performances by training in particular on these talents. As said previously, the capability to quickly building up stimulation may be associated with coordination. it's miles critical to precisely mimic the intermuscular coordination styles of vertical jumping in the course of training [18].

It has been counseled that creating pretension by using co-contractions is the only effective acute approach to lessen muscle slack [19]. training should therefore purpose to enhance the functionality to increase pretension correctly. this can be achieved with the aid of appearing actions beneath time stress and applying volatile masses and surfaces for the duration of schooling [20]. Especially, the athlete does now not have time to carry out a massive countermovement while there's time pressure. therefore, pretension is the simplest realistic solution to minimize muscle slack and entire the motion underneath time strain. unstable hundreds and surfaces may also reason perturbations inside the route, and while there is very little time for correction of these perturbations, reflexes can be too gradual. Pretension is the simplest adequate approach to minimize those perturbations because of the reflex effect [21].

Individuals might also carry out more motor tasks constantly, and therefore higher jump performance can 
be anticipated for professional as compared to unskilled contributors [23]. Further, youngsters and non-lively people may additionally show greater massive variability in acting motor responsibilities. hence the perfect desire of jumping technique for performance assessments in this populace may be extra important [22]. Records on the variety of leaping overall performance within the counter-movement of jumping with a swing of the arm and the akimbo arm concerning special hobby degrees is missing. but, this records is essential for hints at the favored jumping technique for performance assessments in individuals from various sports activities.

\section{CONCLUSION}

In this study, lower body strength results were obtained using the countermovement jump (C.M.J.) test. The analysis description shows that the Countermovement Jump (C.M.J.) test includes the test components, namely Jump height, Power, Eccentric Force, and Concentric Force. The maximum value of each test component is the jump height 43.7 , power 4280 , concentric force 1155 , eccentric force 789.

Research on lower body strength conducted by kickboxing athletes, also known as Countermovement Jump, has proven to be reliable compared to other jumping tests. Therefore C.M.J. can be a suitable test for athletes who follow sports that require high levels of explosive power.

\section{ACKNOWLEDGMENTS}

Thanks to KineticX Lab, Rumble TC, all parties and respondents in this research are willing to help researchers make the research smooth.

\section{REFERENCES}

[1] Claudino, J. G., Cronin, J., Mezêncio, B., McMaster, D. T., McGuigan, M., Tricoli, V., Amadio, A. C., \& Serrão, J. C. The countermovement jumps to monitor neuromuscular status: A meta-analysis. Journal of Science and Medicine in Sport, 20(4), 397-402. https://doi.org/10.1016/j.jsams.2016.08.011. (2017).

[2] Laffaye, G., Wagner, P. P., \& Tombleson, T. I. L. Countermovement jump height: Gender and sportspecific differences in the force-time variables. Journal of Strength and Conditioning Research, 28(4), 1096-1105. https://doi.org/10.1519/JSC.0b013e3182a1db03. (2014).

[3] McErlain-Naylor, S., King, M., \& Pain, M. T. home. G. Determinants of countermovement jump performance: a kinetic and kinematic analysis. Journal of Sports Sciences, 32(19), 1805-1812. https://doi.org/10.1080/02640414.2014.924055. (2014).
[4] McMahon, J. J., Lake, J. P., Dos'Santos, T., Jones, P. A., Thomasson, M. L., \& Comfort, P. Countermovement Jump Standards in Rugby League. Journal of Strength and Conditioning Research, Publish Ah(July). https://doi.org/10.1519/jsc.0000000000003697. (2020).

[5] McMahon, J. J., Suchomel, T. J., Lake, J. P., \& Comfort, P. Understanding the critical phases of the countermovement jump force-time curve. Strength and Conditioning Journal, 40(4), 96-106. https://doi.org/10.1519/SSC.0000000000000375. (2018).

[6] Richter, A., Räpple, S., Kurz, G., \& Schwameder, H. Countermovement jump in performance diagnostics: Use of the correct jumping technique. European Journal of Sport Science, 12(3), 231237.

https://doi.org/10.1080/17461391.2011.566369. (2012).

[7] Ruddock, A. D., Wilson, D. C., Thompson, S. W., Hembrough, D., \& Winter, E. M. Strength and Conditioning for Professional Boxing. Strength \& Conditioning Journal, 38(3), 81-90. https://doi.org/10.1519/ssc.0000000000000217. (2016).

[8] Van Hooren, B., \& Zolotarjova, J.. The Difference between Countermovement and Squat Jump Performances: A Review of Underlying Mechanisms with Practical Applications. Journal of Strength and Conditioning Research, 31(7), 20112020.

https://doi.org/10.1519/JSC.0000000000001913. (2017)

[9] Markström, Jonas L. Olsson, Carl Johan. Is countermovement jump peak force relative to body weight and jump height as predictors for sprint running performances:(in)homogeneity of track and field athletes?. Journal of Strength and Conditioning Research, 27(4), 944-953. 10.1519/JSC.0b013e318260edad. (2013)

[10] James L. Nuzzo,Jonathan H. Anning, And Jessica M. Scharfenberg. The Reliability Of Three Devices Used For Measuring Vertical Jump Height. Journal Of Strength And Conditioning Research. 2580 2590. (2013).

[11] Balsalobre-Fernandez C, Tejero-Gonzalez CM, del Campo-Vecino J. Relationships between training load, salivary cortisol responses and performance during season training in middle and long-distance runners. PLoS One; 9(8). (2014)

[12] Balsalobre-Fernandez C, Tejero-Gonzalez CM, del Campo-Vecino J. Hormonaland neuromuscular responses to high-level middle- and long-distance compe-tition. Int $\mathrm{J}$ Sports Physiol Perform; 9(5):839-844.3. (2014)

[13] Gathercole RJ, Stellingwerff T, Sporer BC. Effect of acute fatigue and training adaptation on countermovement jump performance in elite 
snowboard cross athletes. J Strength Cond Res; 29(1):37-46. (2015)

[14] Arcos AL, Yanci J, Mendiguchia J et al. Short-term training effects of vertically and horizontally oriented exercises on neuromuscular performance in professional soccer players. Int J Sports Physiol Perform; 9(3):480-488. (2014)

[15] About A, Chtourou H, Hammouda O et al. Effects of Ramadan on the diurnal variations of physical performance and perceived exertion in adolescent soccer players. Biol Rhythm Res; 44(6):869-875. (2013)

[16] About A, Chtourou H, Masmoudi L et al. Effects of Ramadan fasting on male judokas performances in specific and non-specific judo tasks. Biol Rhythm Res; 44(4):645-654.54. Anastasi SM, Hamzeh MA. (2013)

[17] Blache, Y., \& Monteil, K... Effect of arm swing on adequate energy during vertical jumping: Experimental and simulation study. Scandinavian Journal of Medicine \& Science in Sports, 23, 121129. (2013)

[18] Tillin, N.A., Pain, M.T.G., \& Folland, J.P. Shortterm training for explosive strength causes neural and mechanical adaptations. Experimental Physiology, 97, 630-641. (2012).
[19] Tillin, N.A., Pain, M.T.G., \& Folland, J. Explosive force production during isometric squats correlates with athletic performance in rugby union players. Journal of Sports Sciences, 31, 66-76. (2013).

[20] Blache, Y., \& Monteil, K. Effect of arm swing on adequate energy during vertical jumping: Experimental and simulation study. Scandinavian Journal of Medicine \& Science in Sports, 23, 121129. (2013)

[21] McLellan, C.P., Lovell, D.I., \& Gass, G.C. The role of rate of force development on vertical jump performance. Journal of Strength and Conditioning Research, 25, 379-385. (2011)

[22] Owen NJ, Watkins J, Kilduff LP, Bevan HR, and Bennett MA. Development of a Criterion Method to Determine Peak Mechanical Power Output in a Countermovement Jump. J Strength Cond Res 28: 1552-1558, (2014).

[23] Gathercole R, Sporer B, Stellingwerff T, and Sleivert G. Alternative Countermovement-Jump Analysis to Quantify Acute Neuromuscular Fatigue. Int J Sports Physiol Perform 10: 84-92. (2015).

[24] Gutierrez-Davila M, Amaro FJ, Garrido JM, and Rojas FJ. An analysis of two styles of arm action in the vertical countermovement jump. Sports Biomech 13: 135-143. (2014). 